\title{
COMMISSIONING OF THE SUPERCONDUCTING RF CAVITIES FOR THE CESR LUMINOSITY UPGRADE *
}

\author{
$\underline{\text { S. Belomestnykh }}{ }^{\#}$, P. Barnes, E. Chojnacki, R. Ehrlich, R. Geng ${ }^{+}$, D. Hartill, R. Kaplan, \\ J. Knobloch, E. Nordberg, H. Padamsee, S. Peck, P. Quigley, J. Reilley, D. Rubin, J. Sears, \\ V. Veshcherevich, Laboratory of Nuclear Studies, Cornell University, Ithaca, NY 14853, U.S.A.
}

\begin{abstract}
The new superconducting RF system consisting of four single-cell cavity modules is an important part of the CESR Luminosity Upgrade. We describe the commissioning of the first three accelerating modules. This includes in situ testing and conditioning, pulsed power and beam processing of RF windows, commissioning of various cryogenic feedback loops, measuring cavity spacing and phasing with beam, and high-current operation.
\end{abstract}

\section{INTRODUCTION}

Since 1997 the new superconducting RF (SRF) system for the CESR Luminosity Upgrade [1,2] is in the stage of installation and commissioning of cavities one by one. This allows the collider to continue its high energy physics operation with short shutdowns for cavity installations. As it was described in Ref. [1], there are two RF straight sections in CESR, East one and West one, each hosting two single-cell cavity cryomodules. Correspondingly, cavities are called E1 and E2, W1 and W2. Each cavity pair is fed by RF power from one klystron via WR1800 waveguide and an RF power splitter (magic $\mathrm{T}$ or hybrid) and by cryogen liquids via a station cryogen distribution box.

The first of four cavities, E2, was the first superconducting HOM damped cavity in the world installed for a long-term operation. Initially, its performance with beam was limited by multipacting in the ceramic window and waveguide region of cavity RF coupler [3] in travelling wave regime. This limitation was eventually overcome after using several techniques of in situ processing, and after several cavity warm ups to room temperature. Obtained experience and understanding of the nature of limitations allowed us to introduce several important modifications to the cryomodule design and preparation procedures. After that, installation of the second module, E1, in October'98 and its subsequent commissioning was significantly easier. W1 cavity is installed in CESR during February-March'99 shutdown, and is being commissioned. W2 cryomodule is being assembled and is scheduled for installation in summer.

"Work supported by the National Science Foundation.

"Email: sab@1ns62.lns.cornell.edu

${ }^{+}$On leave from the IHIP, Peking University, Beijing 100871, PRC.

\section{ACCELERATING MODULE PREPARATION AND TESTS}

Prior to assembly of the superconducting cavity into a horizontal cryostat [4], all major components, such as cryostat, cavity, RF window, and HOM loads, are subjected to acceptance tests. The cryostats are tested at liquid nitrogen temperature with dummy cavity inserts. Upon receiving from a manufacturer, all $\mathrm{Nb}$ cavities are tested in a vertical cryostat [5]. The cavity preparation procedure includes chemical etching by 1:1:2 $\mathrm{BCP}$ acid mix with the acid temperature below $15^{\circ} \mathrm{C}$, then highpressure rinsing and drying in a class 10 clean room. All high vacuum components are assembled and leak checked in the clean room, then vacuum baked. RF windows are processed in pairs in standing wave mode to $125 \mathrm{~kW}$ and in travelling wave mode up to $450 \mathrm{~kW} \mathrm{CW}$ [3]. After assembly is complete, a cryomodule (Figure 1) must pass a final high power acceptance test. In different tests our four cavities reached maximum accelerating gradients of $12 \mathrm{MV} / \mathrm{m}, 10 \mathrm{MV} / \mathrm{m}, 11$ $\mathrm{MV} / \mathrm{m}, 7.8 \mathrm{MV} / \mathrm{m}$.

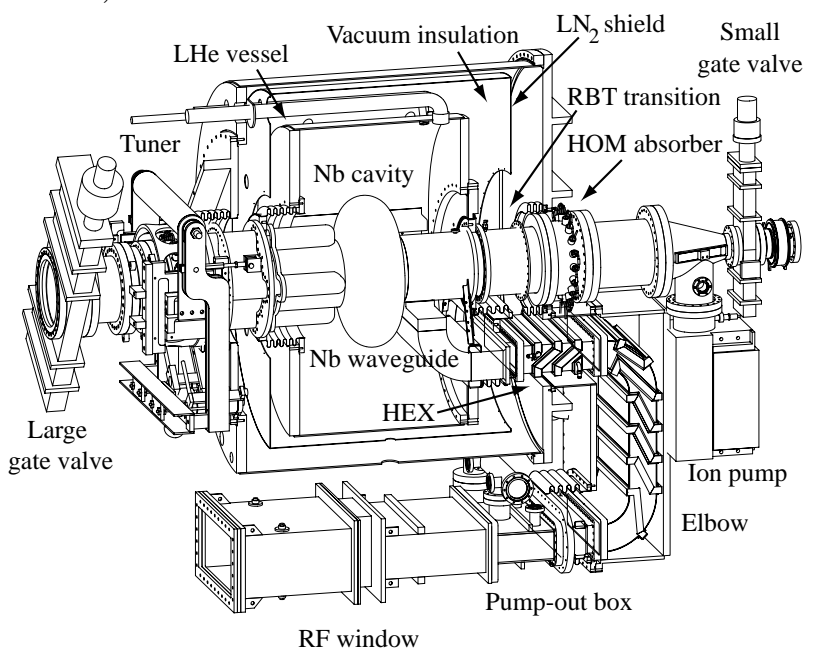

Figure 1: The CESR B-cell cryomodule.

\section{CONTROLS AND DATA ACQUISITION}

Each cryostat has several cryogenic feedback loops. Most important of them are: cryostat liquid helium level, helium bath pressure, and waveguide heat exchanger (HEX) gas flow loops. PID controllers are used in all feedback loops. The station cryogen distribution box 
contains the supply and return control valves, which are used to regulate the level and bath pressure correspondingly. The helium level is kept constant within $\pm 1 \%$, and the pressure is kept constant within \pm 0.02 psi. The waveguide HEX is a short section of vacuum waveguide cooled by cold helium gas borrowed from the cryostat (Figure 1). A feedback loop keeps HEX gas flow constant by regulating a gas flow valve installed in the HEX return line.

In addition to incorporating SRF into the CESR data acquisition system, a new graphics display system, based on the "little language" GDL (Graphics Display Language) developed by S. Peck, is used to show data from the database on an X-terminal [6]. The GDL provides such features to display signals and parameters as labelled schematics, strip charts, meters, and tables. These features allow easy access to data and straight forward interface between users and sensors.

\section{OPERATING EXPERIENCE WITH THE FIRST CAVITY}

\subsection{Installation and commissioning}

E2 SRF cavity was installed into CESR storage ring in September'97 in place of one of the 5-cell normalconducting (NRF) copper cavities. At first, E2 cavity operated at a field of $6.3 \mathrm{MV} / \mathrm{m}$. Later the field was raised to $7 \mathrm{MV} / \mathrm{m}$.

The cryomodules have gate valves on both ends. Therefore, we connect them to the machine vacuum chamber under vacuum and they do not require reprocessing from scratch. The base pressure in the cold cryomodule without RF is 3 to $5 \times 10^{-10}$ Torr at the taper and RF window. The pressure rise with RF and beam is about one order of magnitude by the end of a three month CESR running period. After the first cool down, as the beam current increased in CESR, hard vacuum trips occurred in the cavity window region at currents around $230 \mathrm{~mA}$. The vacuum trips occurred when the power in travelling wave, i.e. forward power minus reflected power, was $90 \mathrm{~kW}$. This was a repeatable limit at different beam currents due to gas evolution in the input window/coupler region. We associated those vacuum trips with multipacting in the RF coupler region.

\subsection{RF coupler in situ processing}

To increase the power delivered to beam we explored several processing techniques. RF processing without beam and off cavity resonance with powers up to 160 $\mathrm{kW} \mathrm{CW}$ had essentially no effect on the travelling wave vacuum trip limit.

It is a well known approach to suppress multipacting in coaxial RF windows by applying DC bias electric field on the inner conductor. This external field disturbs trajectories of electrons and changes resonant conditions of multipacting. Depending on strength and direction of the external field, the multipacting can be either suppressed or enhanced. In the case of a rectangular waveguide it is easier to achieve the same goal by using DC magnetic field instead. We used 10 Gauss transverse magnetic field of permanent magnets. This enhanced multipacting in the RF window region and allowed us to "clean" the surface of the ceramics and adjacent waveguide without beam. Later, calculations [7] confirmed that a transverse magnetic field enhances both intensity and bandwidth of multipacting zones. This technique advanced travelling wave limit to $110 \mathrm{~kW}$.

Quite often, especially in case of "weak" multipacting, it is possible to use "beam processing". The beam injection is stopped as soon as window vacuum activity is detected and started again as soon as vacuum is improved. Alternatively, the RF phase between two RF stations can be adjusted to increase or decrease power to the SRF cavity. However, the beam is frequently lost during beam processing, making it slow and inefficient. We use it mainly as a way to check RF power limit after processing with other techniques.

The greatest success so far has been achieved when processing without beam by increasing power in the pulsed mode on or close to cavity resonance. Such processing allows us to process the coupler region in the travelling wave mode. Because the SRF cavity is heavily overcoupled without beam, the emitted power at RF shut-off is four times higher than the incident power. This creates the travelling wave mode for a very short time though. Changing the frequency around resonance shifts the standing wave pattern in the waveguide, and processes its different regions. Above $90-100 \mathrm{~kW}$ forward power, the cavity quenches and becomes close to matched load. At this and higher powers we disable the quench detector and allow the cavity to sit in quench state for entire duration of pulse, typically $10 \mathrm{~ms}$ at $10 \%$ duty cycle. By pulse processing to $150 \mathrm{~kW}$, the beam power could gradually be raised to $140 \mathrm{~kW}$ and beam current to $350 \mathrm{~mA}$. At this power level we observed for a first time dependence of the RF power on the cavity field: transmitted power is higher when there is bigger standing wave component. Computer simulations [7] confirmed that travelling to standing wave mixing ratio affects multipacting bands.

\subsection{Operating experience}

Alongside with normal vacuum actions (multipacting), which we were able to process, other, very fast, vacuum events were observed. Those events were accompanied by temperature rise on the HEX and huge spikes of hydrogen on RGA. We attributed this effect to releasing cryopumped hydrogen due to RF heating of the HEX surface and following arc. The number of fast vacuum trips was increasing with time and eventually they limited maximum RF power trough the coupler. Analysis of the residual gas evolution [8] during cavity warm up showed that during 3 months of operation cold surfaces accumulated up to 7 equivalent monolayers of 
hydrogen. RGA spectra showed different gas species. Most pronounced among them are hydrogen, carbon monoxide, and water vapour.

After warming up the cavity to room temperature and baking the window in situ to $110^{\circ} \mathrm{C}$ during scheduled CESR shutdown, the beam power delivered began to rise steadily and reached $180 \mathrm{~kW}$. After about 2 months in operation, the ability of the cavity to deliver RF power began to deteriorate again due to the fast vacuum trips.

Operating experience obtained with the first SRF cavity allowed us to introduce several changes in cryomodule design and preparation procedure: i) to improve pumping speed near the RF window [9], we redesigned its pumping layout; ii) to lower heat deposition to the HEX wall due to RF losses and to improve cooling, we increased thickness of copper plating from $6.4 \mu \mathrm{m}$ to $25 \mu \mathrm{m}$ and increased helium gas flow through the HEX by a factor of three; iii) we rounded sharp corners in the vacuum waveguide; iv) baking temperature for $\mathrm{RF}$ window ceramics was increased from $110^{\circ} \mathrm{C}$ to $150^{\circ} \mathrm{C}$, following suggestion in [10]; v) all high vacuum components, including copper plated ones, are now thoroughly vacuum baked. We did not bake all of the copper plated parts before, being afraid to damage plating.

\section{HIGH BEAM CURRENT OPERATION}

The second SRF cavity, E1, was installed in the machine in October'99. Its commissioning went very smoothly and we were soon able to reach and exceed $200 \mathrm{~kW}$ power delivered to beam by one cavity. Operating with two SRF cavities proved to be easier and more stable than with one SRF and one NRF cavity in the same pair. The history of RF power delivered to beam by the SRF cavities is shown in Figure 2. We must note that the RF coupler vacuum trips were the main limiting factor for the beam current increase only in the beginning of E2 cavity operation. Lately, as CESR reached total beam current in excess of $500 \mathrm{~mA}$, a longitudinal dipole coupled bunch instability and overheating of some vacuum chamber components became the major limitations [11, 12].

To keep beam loading the same for both cavities, RF voltage equal for electron and positron beams and maintain interaction point in the center of the detector, it is necessary to carefully position cavities along the beam axis and adjust their waveguide feed lengths. This is especially important at high beam currents. The procedure of deducing errors in relative cavity spacing and phasing from RF forward and reflected power measurements with and without beam was developed by D. Morse at CESR (see appendix A in [13]). We use this procedure to verify installation and make fine adjustments when necessary.

One of the most important features of a CESR B-cell cavity is its low beam coupling impedance due to strong HOM damping [14]. Installation of the E2 and E1 SRF cavities increased beam current thresholds of a longitudinal dipole coupled bunch instability in CESR, though the total HOM impedance of the ring is still dominated by remaining NRF cavities [15]. Beaminduced HOM power is dissipated in two ferrite-lined beam pipe absorbers $[16,17]$. The maximum extracted E2 cavity HOM power was about $5.7 \mathrm{~kW}$ at $510 \mathrm{~mA}$ total current in two beams of nine three-bunch trains each. According to calculations [18] two tapers provide a significant portion of the cryomodule loss factor. Therefore, there are no tapers between E1 and E2 cavities.

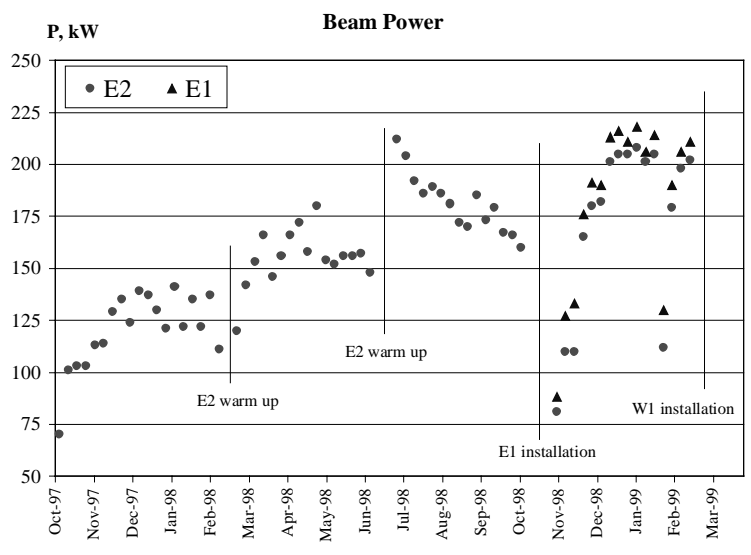

Figure 2: RF power delivered to beam by SRF cavities.

\section{CONCLUSIONS}

Three superconducting cavity modules are installed in CESR so far. Two of them have been successfully commissioned and are in operation. The third module is being commissioned now. The SRF cavities provided very safe and reliable operation with high beam currents up to $550 \mathrm{~mA}$ and allowed CESR to establish a new peak luminosity record of $8 \times 10^{32} \mathrm{~cm}^{-2} \mathrm{~s}^{-1}$ as well as several integrated luminosity records. The fourth cryomodule is being assembled and is scheduled for installation in summer'99. Complete installation of all four HOM damped cavities will reduce ring broad band impedance by $48 \%$ and will provide stable operation of CESR with many bunches at high beam currents.

\section{REFERENCES}

[1] S. Belomestnykh, et al., Proc. 5th EPAC, Vol. 3, pp. 2100-2103.

[2] S. Belomestnykh, et al., Proc. 1997 PAC, Vol. 3, pp. 3075-3077.

[3] E. Chojnacki, et al., Part. Accel., 61, pp. [309-319]/45-55 (1998).

[4] J. Kirchgessner, et al., Proc. 7th SRF Workshop, Vol. 1, pp. 35-37

[5] D. Moffat, et al., Proc. 1993 PAC, Vol. 2, pp. 763-765.

[6] R. Geng and S. Peck, Cornell LNS Report SRF/D980407-01.

[7] R. L. Geng and H. Padamsee, THAL5, these proceedings.

[8] R. L. Geng and H. Padamsee, MOP136, these proceedings.

[9] R. L. Geng and P. Barnes, Cornell LNS Report SRF980227-02.

[10] M. Neubauer, et al., Proc. 5th EPAC, Vol. 3, pp. 2059-2061.

[11] D. L. Rubin, Proc. 6th EPAC, Stockholm, 1998.

[12] A. Temnykh, Proc. XVIIth ICHEA, Dubna, 1998.

[13] S. Belomestnykh, et al., Cornell LNS Report SRF961217-04.

[14] H. Padamsee, et al., Part. Accel., 40, pp. 17-41 (1992).

[15] M. G. Billing and S. Belomestnykh, TUA18, these proceedings.

[16] S. Belomestnykh, et al., Proc. 1995 PAC, Vol. 5, pp. 3394-3396.

[17] E. Chojnacki, W. J. Alton, MOP77, these proceedings.

[18] S. Belomestnykh, W. Hartung, Report SRF960202-01 (1996). 